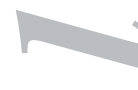

\title{
Complicaciones precoces tras el trasplante hepático. ¿Podemos llegar a predecirlas?
}

El trasplante hepático constituye, en la actualidad y desde hace años, el tratamiento idóneo de la enfermedad hepática terminal, tanto aguda como crónica, tenga o no implantado un hepatocarcinoma.

Desde que Starzl, en 1963, realizara el primer trasplante hepático, y posteriormente en 1983 se reconociera por el Instituto Nacional de la Salud de EE.UU. como una opción terapéutica (1), hasta la actualidad se han producido avances importantes en la indicación, tratamiento y seguimiento de los pacientes sometidos a trasplante hepático.

El mejor conocimiento de la historia natural de la enfermedad hepática, la indicación óptima del trasplante, el desarrollo de la cirugía, los avances logrados en el cuidado postoperatorio, complicaciones postrasplante inmediatas y a largo plazo y el desarrollo de modernos inmunosupresores han permitido un claro incremento, no sólo de la supervivencia de los pacientes trasplantados, sino también de su calidad de vida.

En la actualidad, el cuidadoso y estricto proceso de evaluación pretrasplante pretende detectar las diferentes situaciones de la funcionalidad hepática o de otros órganos y sistemas, que conllevarían un pobre pronóstico postrasplante. Identificar todos estos factores en el pretrasplante supondría no sólo optimizar la viabilidad del injerto hepático, sino mejorar la supervivencia al disminuir la morbilidad.

No todas las complicaciones derivan del receptor sino que pueden determinarse por la situación clínica y otras características del donante, por las complicaciones derivadas de la cirugía y la medicación que se precise en el postrasplante (inmunosupresora, antiinfecciosa, etc.).

Las complicaciones más frecuentes que se producen como consecuencia de lo anteriormente referido son las renales, infecciosas, inmunológicas (rechazo) y neurológicas. Vienen determinadas, en general, por las interacciones existentes entre la situación previa del receptor, del donante y las actuaciones que hay que realizar instrumentalmente o farmacológicamente sobre las complicaciones que se produzcan.

En el presente número de la Revista Española de Enfermedades Digestivas se publica un interesante trabajo de Rodríguez Ariza y cols., titulado Factores pronósticos de complicaciones postoperatorias en el trasplante hepático (2) en el que se evalúan los factores que predicen la aparición en el periodo postrasplante inmediato de cuatro complicaciones potencialmente graves: el desarrollo de insuficiencia renal (IR), la aparición de infecciones, el rechazo agudo del injerto y la existencia de complicaciones neurológicas. Todas ellas pueden condicionar una disminución de la calidad de vida y supervivencia del paciente, por lo que el conocimiento de los factores que predicen su aparición resulta de excepcional interés. 
Una de las más importantes es la insuficiencia renal (IR). Puede relacionarse con la situación pretrasplante, dado que complica muy frecuentemente la enfermedad hepática avanzada, con los cambios hemodinámicos frecuentes e intensos que se producen durante la cirugía y con la necesidad de administrar medicación potencialmente nefrotóxica en el postrasplante para dificultar e idealmente impedir el desarrollo de otras complicaciones, principalmente inmunológicas e infecciosas. A su vez puede tener características de aguda o crónica. La mayor parte de los pacientes desarrollan en el postrasplante inmediato algún grado de IR. Aunque es difícil homogeneizar los resultados, por las diferencias en la definición de IR, su incidencia en las primeras semanas, en relación con los estudios que se evalúen, se sitúa entre el 48 y $94 \%$ (3).

El incremento observado durante los últimos años viene determinado por la utilización cada vez más frecuente de donantes no óptimos, debido al incremento de pacientes en lista de espera, a lo que se añade la realización del trasplante en pacientes con mayor deterioro de la función hepática como consecuencia de la estancia en lista de espera. Esta situación favorece el desarrollo de deterioro de la función renal como consecuencia en gran medida del síndrome hepatorrenal.

Estas condiciones favorecen el desarrollo de alteraciones hemodinámicas durante la cirugía, el aumento de su duración, el incremento de infecciones y finalmente la aparición postrasplante de malfunción del injerto. Todo ello facilita en última instancia el desarrollo de IR.

Las causas más comunes de IR postrasplante son el desarrollo de necrosis tubular aguda y la producción de nefrotoxicidad secundaria a fármacos, fundamentalmente producida por los inmunosupresores inhibidores de la calcineurina (4). En este sentido, en un reciente trabajo español, cuyo objetivo era evidenciar factores de predicción de IR aguda en el primer mes postrasplante, Cabezuelo y cols. (5) observaron cómo en la primera semana postrasplante la IR predominante era la debida a necrosis tubular aguda y la de tipo multifactorial. La relacionada con nefrotoxicidad por ciclosporina aparecía, dado que se relacionaba con su administración y niveles séricos, a partir de la segunda semana. En la publicación de Rodríguez-Ariza y cols. (2), se comprueba, mediante análisis multivariado, cómo la situación renal previa al trasplante, determinada por la concentración sérica de creatinina y el nivel de estrés oxidativo, medido por la concentración de malondialdehído, se relacionan con el desarrollo de IR precoz. Son múltiples las publicaciones en las que se estudia la influencia de los valores de creatinina pretrasplante en el desarrollo de IR en el postrasplante inmediato, encontrando unas influencia en su aparición $(6,7)$, aunque otras no (8).

En la publicación de Cabezuelo y cols. (5), el desarrollo de IR postrasplante se relacionó con la existencia de IR previa al trasplante en su globalidad, aunque no con la cifra de creatinina de forma aislada, junto con la cifra de albúmina, la necesidad de dopamina y la existencia de disfunción del injerto.

Es indudable la gran importancia que tiene el diferenciar en el pretrasplante entre procesos renales parenquimatosos, y por lo tanto mantenidos en su evolución, y los funcionales, relacionados con la existencia de un deterioro marcado de la hepatopatía por la que se indicó el trasplante. Hubiera sido de gran interés que esta información estuviera reflejada en la publicación de Rodríguez Ariza y cols. (2) por la diferente evolución y pronóstico que suponen en la necesidad de depuración extracorpórea o incluso en la indicación de un trasplante renal. Igualmente conocer el porcentaje de pacientes con IR pretrasplante hubiera permitido averiguar el impacto de esta complicación en el postoperatorio inmediato. 
Según publicaciones recientes, la necesidad de depuración extracorpórea tras el trasplante hepático se ha incrementando en la última década, relacionándose con la mayor evolución de la enfermedad hepática y la mayor edad de los receptores, alcanzando entre el 8-17\% (9). Otros autores han confirmado estos hallazgos, al comprobar cómo una cifra de creatinina sérica pretrasplante mayor de $1,9 \mathrm{mg} / \mathrm{dl}$ y un BUN mayor de $27 \mathrm{mg} / \mathrm{dl}$, junto con una estancia en UCI superior a tres días y una puntuación MELD mayor de 21, predecían la necesidad de depuración extracorpórea (10).

Otro de los mecanismos de desarrollo de IR en el postrasplante es el debido a la administración de inhibidores de la calcineurina. Estos inmunosupresores facilitan la liberación de endotelina-1, por lo que se produce vasoconstricción renal y consecuentemente disminución del flujo vascular y desarrollo de IR (11). Son numerosos los estudios, similares al de Rodríguez Ariza y cols. (2), en los que se comprueba cómo el desarrollo de IR postrasplante no se relaciona con la administración de ciclosporina o tacrolimus (12). Estos dos inmunosupresores son la primera opción en el trasplante hepático y lógicamente no deben existir diferencias entre ellos en el desarrollo de IR, dado que ambos pertenecen a la misma familia farmacológica.

Cuando existe insuficiencia renal pretrasplante se han diseñado protocolos de inmunosupresión en los que se comienza con anticuerpos anti-IL2 o micofenolato y corticoides, introduciéndose tardía y lentamente los anticalcineurínicos. Es seguro que, aunque no se hace referencia a ello, en el trabajo que nos ocupa se han utilizado estas variaciones inmunosupresoras en los pacientes con IR pretrasplante.

La búsqueda y prevención de la insuficiencia renal precoz en los pacientes trasplantados es de gran trascendencia, dado que influye de forma importante en su supervivencia. En el trabajo de Fraley y cols. (13) se apreció una mortalidad significativamente mayor en los pacientes con función renal normal previa al trasplante que desarrollaban IR precoz posterior comparado con los que no la desarrollaban (41 vs. $5 \%$ ). Similar fue la observación de Cabezuelo y cols. (5) (16,7 vs. 3,8\% respectivamente).

Otra de las complicaciones importantes del trasplante hepático son las infecciones. En los últimos años, gracias a la instauración de protocolos de profilaxis antibiótica, se ha logrado disminuir la mortalidad que producían inicialmente. En el momento actual, al igual que se comentaba con la IR, la priorización del trasplante basada en el sistema MELD hace que la indicación se realice en pacientes con hepatopatía evolucionada y por lo tanto con mayor inmunocompromiso, mayor necesidad, en general, de transfusión de hemoderivados y mayor duración de la cirugía y estancia en UCI, a lo que posteriormente se añade la administración de inmunosupresores. Todo lo anterior favorece la aparición de infecciones en el postrasplante inmediato.

En este sentido, durante el primer mes tras el trasplante, las infecciones habitualmente están relacionadas con complicaciones quirúrgicas o derivadas de la propia malfunción del injerto, junto con la existencia de periodos prolongados de hospitalización previos. Es en este periodo cuando aparecen la mayoría de las infecciones bacterianas y fúngicas. El desarrollo de infecciones en estos pacientes es mayor que en otro tipo de trasplantes dada la complejidad técnica del trasplante hepático y tratarse de una cirugía abdominal y por lo tanto potencialmente contaminada (14).

Debido a la utilización generalizada de descontaminación intestinal selectiva con quinolonas y antifúngicos, se ha comprobado un cambio en el aislamiento bacteriano (15). En la actualidad el aislamiento predominante es el de cocos gram positivos en contraposición de los bacilos aerobios gram negativos. En un trabajo reciente de 
Losada y cols. (16) en el que se estudiaba la incidencia, microbiología y factores de riesgo de infección precoz en el trasplante hepático, se comprobó una incidencia global del $73,1 \%$, presentándose la mayoría de los episodios infecciosos en los primeros 30 días postrasplante. En el trabajo motivo de este editorial, Rodríguez-Ariza y cols. (2) evidencian una menor incidencia (41\%), posiblemente debido a que consideran infección sólo cuando ha existido un aislamiento microbiológico y no lo hacen ante episodios clínicos sugestivos que responden a la administración empírica de antibióticos, aunque no se haya producido aislamiento. En esta fase precoz del trasplante predominan las infecciones bacterianas tal y como comunican diversos autores $(16,17)$.

El elevado porcentaje de infecciones postrasplante implica una desfavorable supervivencia de los pacientes, dadas las interacciones que suponen sobre los diferentes órganos y sistemas, principalmente el renal. La identificación de factores de riesgo de infecciones postrasplante es de suma importancia para prevenir su aparición y mejorar el pronóstico de estos pacientes. En el trabajo ya referido (2) se identifican como factores de riesgo independientes la existencia de una hemoglobina en el pretrasplante $<10 \mathrm{~g} / \mathrm{dl}$ y una necesidad transfusional de plaquetas de más de 8 unidades. De nuevo estos datos nos indican la influencia de la situación del paciente previa a la cirugía y la duración de esta. Además ambos datos son, en general, consecuencia del grado de hipertensión portal existente y por lo tanto de la evolución de la enfermedad hepática. Son varias las publicaciones en las que se demuestra una clara relación entre la necesidad de administración de hemoderivados y el riesgo de infecciones en diversas cirugías $(18,19)$, al igual que en el trasplante hepático $(20,21)$.

También se ha demostrado cómo la politransfusión secundaria a hemorragia durante la cirugía y en ocasiones el shock acompañante producen una reducción de la actividad de las células natural killer (22) y alteración en la función de los linfocitos $\mathrm{T}$, así como un incremento de la translocación bacteriana y bacteriemia (23), ya favorecida por la propia hepatopatía. Todo ello implica una inmunosupresión natural en el postoperatorio de estos pacientes, que incrementa el riesgo de infecciones bacterianas. Además de lo anterior referido, la transfusión de plaquetas durante el trasplante ha demostrado ser factor predictivo independiente de mortalidad a 1 y 5 años en un reciente trabajo (24).

Otra de las complicaciones frecuentes tras el trasplante es el rechazo agudo, presentando una incidencia muy variable. Su detección precoz es de gran importancia dado el riesgo que implica para el injerto. La máxima incidencia se produce generalmente en las primeras 6 semanas. En el trabajo de Rodríguez-Ariza y cols. (2) se observó en el $37 \%$ de los pacientes, principalmente entre los días 5 y 15 tras el trasplante, con un tiempo medio de aparición de 9 días. Estos datos concuerdan con los obtenidos por diferentes grupos de trasplante (25) con una incidencia del 35,3\%, presentándose en el $93 \%$ en los primeros 45 días, y con un tiempo medio de aparición de 5 días tras el trasplante. Otros autores, en cambio, muestran incidencias mayores $(26,27)$, pudiéndose relacionar con la disparidad de protocolos de inmunosupresión existentes.

La existencia de rechazo agudo se relaciona, como es lógico, con la inmunosupresión administrada, que ha variado a lo largo de los años y con la realización o no de biopsias hepáticas protocolizadas, lo que ha permitido diagnosticar lesiones histológicas compatibles con rechazo agudo sin expresividad clínica ni analítica. En este sentido se ha demostrado cómo tan sólo el 17\% de los episodios de rechazo son clínicamente significativos (28). 
Poder predecir cuáles son los pacientes con mayor probabilidad de sufrir rechazo agudo permitiría disminuir su aparición, mediante la optimización de la inmunosupresión durante los primeros días del postrasplante. Varios trabajos han intentado identificar estos factores de riesgo, obteniendo resultados dispares. Wiesner y cols. (27), sobre una cohorte consecutiva de 762 trasplantados, observaron cómo las variables que se asociaron de forma independiente con la aparición de rechazo agudo fueron la edad (juventud) del receptor, ausencia de insuficiencia renal, ALT elevada, ausencia de edemas previa al trasplante, escasa concordancia HLA, tiempo de isquemia fría mayor de 15 horas y edad del donante (mayor de 30 años). La relación del rechazo con la juventud del receptor coincide con otros estudios $(25,29)$, relacionándose con la disminución de la respuesta inmune que se produce con la edad, que implica también un mayor riesgo de infecciones. En el estudio de RodríguezAriza y cols. (2) no se encuentra relación con la etiología de la hepatopatía, aunque, según se deriva de otros trabajos, parece existir una influencia de la etiología metabólica y autoinmune sobre la alcohólica, en la que la incidencia de rechazo está disminuida (25,30). La explicación podría estar en la persistencia, tras el trasplante, de un sistema inmune hiperactivo en las primeras etiologías y un inmunocompromiso marcado en los de etiología etílica, en relación al efecto tóxico del alcohol y la importante malnutrición que padecen habitualmente estos pacientes. Existen publicaciones en las que se ha establecido una relación entre la infección por el virus de la hepatitis $\mathrm{C}$ y una mayor incidencia de rechazo (26).

Rodríguez-Ariza y cols. (2) comprueban cómo la bilirrubina pretrasplante puede predecir la aparición de rechazo agudo, siendo expresión de la funcionalidad hepática. Los pacientes con buena función hepática (grado A de Child-Pugh) y por tanto con bilirrubina sérica normal o levemente elevada tienen mayor riesgo de sufrir episodios de rechazo. De forma similar, Gómez-Manero y cols. (25) observan cómo los pacientes con hepatopatías más evolucionadas presentan menor riesgo de rechazo como consecuencia de la mayor inmunosupresión propia de la enfermedad, colaborando en esto último el deficiente estado nutricional existente en la enfermedad hepática terminal (31).

Finalmente, otras complicaciones de gran trascendencia tras el trasplante hepático son las neurológicas. Su etiología es múltiple y clínicamente varían desde enfermedades leves a potencialmente mortales. Su incidencia no está bien establecida, dada la heterogeneidad de patologías que abarcan. Aproximadamente entre el 13 y el $43 \%$ de los pacientes trasplantados hepáticos sufren trastornos neurológicos (32$34)$. En el trabajo de Rodríguez-Ariza y cols. (2) la incidencia es del 22\%, similar a la observada por otros autores (34-37), aunque el tipo de complicaciones varía notablemente. Las convulsiones son la complicación más frecuente en el presente trabajo (2), apareciendo aproximadamente en el $16 \%$ de los pacientes. Estos datos contrastan con los obtenidos en otros estudios en los que la encefalopatía, de diferente origen, es la causa principal y las convulsiones ocurren en un menor porcentaje. Saner y cols. (37) observan una incidencia de encefalopatía del 18,5\% y de convulsiones de tan sólo un 5,4\%. Porcentajes similares obtienen Lewis y cols. (34) (11 y 6\%, respectivamente) y Bronster y cols. (35) (11,8 y $8,2 \%$, respectivamente).

Los mecanismos que pueden explicar esta encefalopatía difusa no están aclarados, aunque en un estudio prospectivo español la etiología séptica, anóxica y metabólica explicaron la encefalopatía en la mayoría de los casos, siendo de hecho también la complicación neurológica más frecuente tras el trasplante (15\%) (33). Aunque en algún trabajo se observaba una influencia de la etiología alcohólica y de la cirrosis biliar primaria como factores favorecedores de complicaciones neuroló- 
gicas $(34)$, no se ha confirmado en otras series $(35,37)$. No parece que se haya observado influencia de la inmunosupresión, cuando se realiza con ciclosporina o tacrolimus, en el desarrollo de complicaciones neurológicas en las diferentes publicaciones realizadas, al igual que ha evidenciado la de Rodríguez-Ariza y cols. (2). En el año 1994, Mueller y cols. (38) comprobaron cómo la incidencia de neurotoxicidad moderada o grave era superior en los pacientes tratados con tacrolimus tanto en el postrasplante inmediato como en el tardío y en el retrasplante, aunque el análisis multivariado demostraba también la influencia de parámetros séricos, por lo que las complicaciones neurológicas tendrían un origen multifactorial. Las alteraciones iónicas a nivel sérico como la hipo- e hipernatremia, previa a la realización del trasplante, parece favorecer en algunas publicaciones el desarrollo de complicaciones neurológicas (39).

Estas complicaciones neurológicas implican en algunas publicaciones una clara alteración de la supervivencia (34), mientras que en otros la influencia es mínima o nula (37). Finalmente, es interesante reseñar cómo la realización de trasplante con donante vivo presenta una menor incidencia de alteraciones neurológicas que los pacientes trasplantados con donante cadáver (40).

Son varias las determinaciones de citoquinas y otros marcadores que se estudian en el trabajo de Rodríguez-Ariza y cols. (2) encontrándose relaciones interesantes, y quizás en el futuro de utilización, en la demostración de posibles alteraciones como la insuficiencia renal o las complicaciones neurológicas. En la actualidad su uso parece exclusivamente reservado al campo de la investigación, dada la dificultad de determinarlas en la práctica clínica habitual.

Finalmente, hay que reseñar que estudios como el realizado y publicado en el presente número de la Revista Española de Enfermedades Digestivas, aunque deben ser tomados con cautela en los resultados por el escaso número de pacientes analizados, son de gran importancia porque intentan clarificar problemas relacionados, en este caso, con complicaciones concretas del paciente trasplantado al que de forma habitual han de enfrentarse los hepatólogos.

Los interesantes resultados obtenidos aconsejan la realización de estudios prospectivos con inclusión de gran número de pacientes y con la colaboración de diferentes centros. A pesar de que queda un importante camino por recorrer, trabajos como el de Rodríguez-Ariza y cols. (2) nos animan a continuar desvelando interrogantes y demostrando el gran potencial investigador de la hepatología de nuestro país.

G. Clemente Ricote y A. Díaz Sánchez

Servicio de Digestivo. Hospital General Universitario Gregorio Marañón. Madrid

\section{BIBLIOGRAFÍA}

1. National Institutes of Health. Consensus Development Conference Statement: Liver transplantation, June 2023, 1983. Hepatology 1984; 4: S107-10.

2. Rodríguez-Ariza A, Monrobel A, Martínez-Galisteo E, Alicia Padilla C, Bárcena JA, Fraga E, et al. Factores pronósticos de complicaciones postoperatorias en el trasplante hepático. Rev Esp Enferm Dig 2008; 100 (3): 129-38.

3. Yalavarthy R, Edelstein CL, Teitelbaum I. Acute renal failure and chronic kidney disease following liver transplantation. Hemodial Int 2007; 11: S7-12.

4. Vitko S, Viklicky O. Cyclosporine renal dysfunction. Transplant Proc 2004; 36 (Supl. 2): S243-7. 
5. Cabezuelo JB, Ramírez P, Ríos A, Acosta F, Torres D, Sansano T, et al. Risk factors of acute renal failure after liver transplantation. Kidney Int 2006; 69: 1073-80.

6. Bilbao I, Charco R, Balsells J, Lázaro JL, Hidalgo E, Llopart L, et al. Risk factors for acute renal failure requiring dialysis after liver transplantation. Clin Transplant 1998; 12: 123-9.

7. Lima EQ, Zanetta DM, Castro I, Massarollo PC, Mies S, Machado MM, et al. Risk factors for development of acute renal failure alter liver transplantation. Ren Fail 2003; 25: 553-60.

8. Nuño J, Cuervas-Mons V, Vicente E, Turrión V, Pereira F, Mora NP, et al. Renal failure after liver transplantation: Analysis of risk factors in 139 liver transplant recipients. Transplant Proc 1995; 27: 2319-20.

9. Gonwa TA, Mai ML, Melton LB, Hays SR, Goldstein RM, Levy MF, et al. End-stage renal disease (ESRD) after orthotopic liver transplantation (OLTX) using calcineurin-based immunotherapy: Risk of development and treatment. Transplantation 2001; 72: 1934-9.

10. Sánchez EQ, Gonwa TA, Levy MF, Goldstein RM, Mai ML, Hays SR, et al. Preoperative and perioperative predictors of the need for renal replacement therapy alter orthotopic liver transplantation. Transplantation 2004; 78: 1048-54

11. Olyaei AJ, de Mattos AM, Bennett WM. Nephrotoxicity of immunosuppressive drugs: New insight and preventive strategies. Curr Opin Crit Care 2001; 7: 384-9.

12. Platz KP, Mueller AR, Blumhardt G, Bachmann S, Bechstein WO, Kahl A, et al. Nephrotoxicity alter orthotopic liver transplantation in cyclosporin A and FK 506-treated patients. Transpl Int 1994; 7 (Supl. 1): S52-7.

13. Fraley DS, Burr R, Bernardini J, Angus D, Kramer DJ, Jonson JP. Impact of acute renal failure on mortality in end-stage liver disease with or without transplantation. Kidney Int 1998; 54: 518-24.

14. Winston DJ, Emmanouilides C, Busuttil RW. Infections in liver transplant recipients. Clin Infect Dis 1995; 21: 1077-91.

15. Gorensek MJ, Carey WD, Washington JA, Vogt DP, Brougham TA, Westveer MK. Selective bowel decontamination with quinolones and nystatin reduces gram-negative and fungal infections in orthotopic liver transplant recipients. Cleve Clin J Med 1993; 60: 139-44.

16. Losada I, Cuervas-Mons V, Millán I, Damaso D. Infección precoz en el paciente con trasplante hepático: incidencia, gravedad, factores de riesgo y sensibilidad antibiótica de los aislados bacterianos. Enferm Infecc Microbiol Clin 2002; 20: 422-30.

17. García S, Roque J, Ruza F, González M, Madero R, Alvarado F, et al. Infection and associated risk factors in the immediate postoperative period of pediatric liver transplantation: A study of 176 transplants. Clin Transplant 1998; 12: 190-7.

18. Chang H, Hall GA, Geerts WH, Greenwood C, McLeod RS, Sher GD. Allogenic red blood cell transfusion is an independent risk factor for the development of postoperative bacterial infection. Vox Sang 2000; 78: 13-8

19. Hill GE, Frawley WH, Griffith KE, Forestner JE, Miney JP. Allogenic blood transfusion increases the risk of postoperative bacterial infection: A meta-analysis. J Trauma 2003; 54: 908-14.

20. Palomo-Sánchez JC, Jiménez C, Moreno-González E, García I, Palma F, Loinaz C, et al. Effects of intraoperative blood transfusion on postoperative complications and survival after orthotopic liver transplantation. Hepatogastroenterology 1998; 45: 1026-33.

21. Mor E, Jennings L, Gonwa TA, Holman MJ, Gibbs J, Solomon H, et al. The impact of operative bleeding on outcome in transplantation of the liver. Surg Gynecol Obstet 1993; 176: 219-27.

22. Gascon P, Zoumbos NC, Young NS. Immunologic abnormalities in patients receiving multiple blood transfusions. Ann Intern Med 1984; 100: 173-7.

23. Rush BF, Sori AJ, Murphy TF, Smith S, Flanagan JJ, Machiedo GW. Endotoxemia and bacteriemia during hemorrhagic shock. Ann Surg 1988; 207: 549-54.

24. De Boer MT, Christensen MC, Asmussen M, Van der Hilst CS, Hendriks HGD, Slooff MJH, et al. The impact of intraoperative transfusion of platelets and red blood cells on survival after liver transplantation Anesth Analg 2008; 106: 32-44.

25. Gómez-Manero N, Herrero JI, Quiroga J, Sangro B, Pardo F, Cienfuegos JA, et al. Prognostic model for early acute rejection after liver transplantation. Liver Transpl 2001; 7: 246-54.

26. McTaggart RA, Terrault NA, Vardanian AJ, Bostrom A, Feng S. Hepatitis C etiology of liver disease is strongly associated with early acute rejection following liver transplantation. Liver Transpl 2004; 10: 975-8.

27. Wiesner RH, Demetris AJ, Belle SH, Seaberg EC, Lake JR, Zetterman RK, et al. Acute hepatic allograft rejection: Incidence, risk factors, and impact on outcome. Hepatology 1998; 28: 638-45.

28. Tippner C, Nashan B, Hoshino K, Schmidt-Sandte E, Akimaru K, Boker KH, et al. Clinical and subclinical acute rejection early after liver transplantation: Contributing factors and relevance for the long-term course. Transplantation 2001; 72: 1122-8.

29. Mor E, Solomon H, Gibbs JF, Holman MJ, Goldstein RM, Husberg BS, et al. Acute cellular rejection following liver transplantation: Clinical pathologic features and effect on outcome. Semin Liver Dis 1992; 12: 28 40 .

30. Hayashi M, Keeffe EB, Krams SM, Martínez OM, Ojogho ON, So SKS, et al. Allograft rejection after liver transplantation for autoinmune liver diseases. Liver Transpl Surg 1998; 4: 208-14.

31. Bathgate A, Hynd P, Sommerville D, Hayes P. The prediction of acute cellular rejection in orthotopic liver transplantation. Liver Transpl Surg 1999; 5: 475-9.

32. Neuhaus P, McMaster P, Calne R, Pichlmayr R, Otto G, Williams R, et al. Neurological complications in THO European multicentre study of FK 506 and cyclosporin in primary liver transplantation. Transpl Int 1994; 7 (Supl. 1): S27-31.

33. Pujol A, Graus F, Rimola A, Beltrán J, García Valdecasas JC, Navasa M, et al. Predictive factors of in-hospital CNS complications following liver transplantation. Neurology 1994; 44: 1226-30.

34. Lewis MB, Howdle PD. Neurologic complications of liver transplantation in adults. Neurology 2003; 61: 1174-8.

35. Bronster DJ, Emre S, Boccagni P, Sheiner PA, Schwartz ME, Miller CM. Central nervous system complications in liver transplant recipients: Incidence, timing, and long-term follow-up. Clin Transplant 2000; 14: 1-7. 
36. Stein DP, Lederman RJ, Vogt DP, Carey WD, Broughan TA. Neurological complications following liver transplantation. Ann Neurol 1992; 31: 644-9.

37. Saner FH, Sotiropoulos GC, Gu Y, Paul A, Radtke A, Gensicke J, et al. Severe neurological events following liver transplantation. Arch Med Res 2007; 38: 75-9.

38. Mueller AR, Platz KP, Bechstein WO, Schattenfroh N, Stoltenburg-Didinger G, Blumhardt G, et al. Neurotoxicity after orthotopic liver transplantation. A comparison between cyclosporine and FK506. Transplantation 1994; 58: 155-70.

39. Londoño MC, Guevara M, Rimola A, Navasa M, Taura P, Mas A, et al. Hyponatremia impairs early posttransplantation outcome in patients with cirrhosis undergoing liver transplantation. Gastroenterology 2006; 130: $1135-43$.

40. Saner FH, Gu Y, Minouchehr S, Ilker K, Fruhauf NR, Paul A, et al. Neurological complications after cadaveric and living donor liver transplantation. J Neurol 2006; 253: 612-7. 